\section{MS12-P7 Crystal transformation using IR laser radiation}

\author{
Breyan H. Ross ${ }^{1,2,3}$, Evi Stahl ${ }^{3}$, Stephan $\mathrm{Krapp}^{2}$, Robert \\ Huber $^{1,3,4,5}$, Reiner A. Kiefersauer ${ }^{1,2}$
}

1. Max-Planck-Institut für Biochemie, Am Klopferspitz 18, 82152 Martinsried, Germany

2. Proteros Biostructures $\mathrm{GmbH}$, Bunsenstrasse 7a, 82152 Martinsried, Germany

3. Technische Universität München, Lichtenbergstrasse 4, 85747 Garching, Germany

4. Zentrum für Medizinische Biotechnologie, Universität Duisburg-Essen, 45117 Essen, Germany

5. School of Biosciences, Cardiff University, Cardiff CF10 3US, Wales

email: breyanross@gmail.com

For solving crystallographic biomolecular structures sometimes crystals simply do not diffract good enough. Post-crystallization treatments aim to overcome this situation by adjusting the environmental variables of the crystal in order to improve the diffraction quality. One strategy used in our laboratory is crystal dehydration by Free Mounting System (FMS) technique (1). Using CO dehydrogenase (CODH) crystals as a model of improvement by dehydration we characterized the use of a novel devise to control relative humidity (rh) based on Infra-red laser (2). Interestingly the dehydration process of $\mathrm{CODH}$ shows two stages, with the last one, the more stable and well diffracting state (3). On the other side, depending on the crystal system, dehydration could causes loss of diffraction, one example is tetragonal crystals of lysozyme. We compare the different states to explain how the transformation occurs. Not just protein crystals can be dehydrated, but also DNA crystals can be subject of this approach. We used origami DNA crystals to study crystal transformation, analyzing several variations of the constructs like different ending of the strands. We found that crystal transformation in the case of improvement is accompained by a cell dimension contraction and the formation of new crystal contacts. In the case of deterioration, there is also a contraction of unit cell dimension but there is a reorganization of crystal contacts keeping the symmetry. Using the laser we can evaluate transformation in terms of kinetics or diffraction quality. Evaluating these crystal systems we aim to explore the structural basis of crystal transformation and also to develop the laser technique to control water content in crystal systems exploring possible advantages over the current relative humidity control techniques.

1.- Kiefersauer R., Than M., Dobbek H., Gremer L., Melero M., Strobl S., Dias J., Soulimane T., Huber R. 2000. J. Appl. Cryst. 33, pp. 1223-1230.

2.- Kiefersauer R., Grandl B., Krapp S., Huber R., 2014. Acta Cryst D, 2014. 70, pp. 1224-1232.

3.- Dobbek H., Gremer L., Kiefersauer R., Huber R., Meyer O. 2002. PNAS Vol.99, pp. 15971-15976

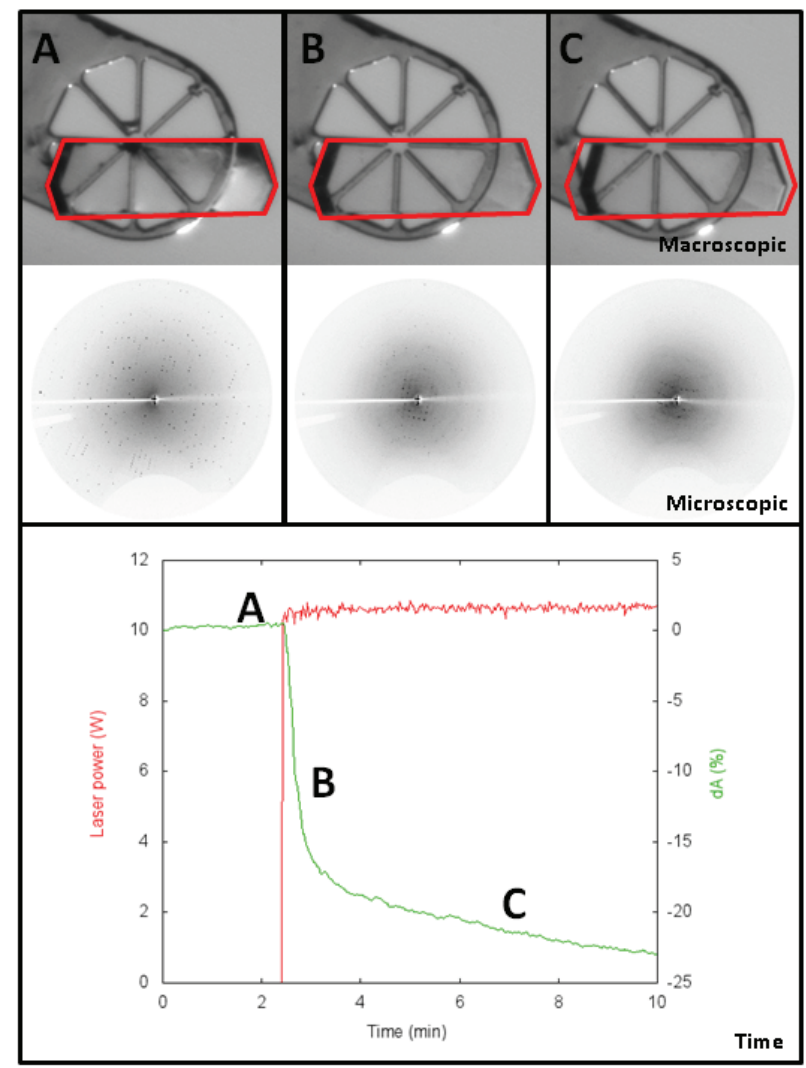

Figure 1. Lysozyme transformation. A, correpond to $96 \%$ rh. B, first intermediate state at $91 \% \mathrm{rh}$. C, final state, below $91 \% \mathrm{rh}$. Lower panel, shows the curve of dehydration measured by back projections of the red square in the upper panel. Constant laser power of $5 \mathrm{~W}$ and increasing frequency up to $1000 \mathrm{~Hz}$.

Keywords: Crystal transformation, infrared laser, dehydration, crystal contacts, crystal improvement, annealing 